Draft VERsion SEPTEMber 22, 2018

Preprint typeset using $\mathrm{AT}_{\mathrm{E} X}$ style emulateapj v. 11/10/09

\title{
A NEW STATISTIC FOR ANALYZING BARYON ACOUSTIC OSCILLATIONS
}

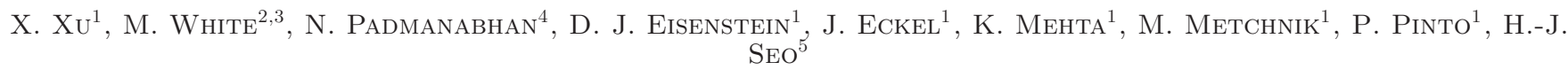 \\ Draft version September 22, 2018
}

\begin{abstract}
We introduce a new statistic $\omega_{\ell}\left(r_{s}\right)$ for measuring and analyzing large-scale structure and particularly the baryon acoustic oscillations. $\omega_{\ell}\left(r_{s}\right)$ is a band-filtered, configuration space statistic that is easily implemented and has advantages over the traditional power spectrum and correlation function estimators. Unlike these estimators, $\omega_{\ell}\left(r_{s}\right)$ can localize most of the acoustic information into a single dip at the acoustic scale while also avoiding sensitivity to the poorly constrained large scale power (i.e., the integral constraint) through the use of a localized and compensated filter. It is also sensitive to anisotropic clustering through pair counting and does not require any binning of data. We measure the shift in the acoustic peak due to nonlinear effects using the monopole $\omega_{0}\left(r_{s}\right)$ derived from subsampled dark matter catalogues as well as from mock galaxy catalogues created via halo occupation distribution (HOD) modeling. All of these are drawn from 44 realizations of $1024^{3}$ particle dark matter simulations in a $1 h^{-1} \mathrm{Gpc}$ box at $\mathrm{z}=1$. We compare these shifts with those obtained from the power spectrum and conclude that the results agree. We therefore expect that distance measurements obtained from $\omega_{0}\left(r_{s}\right)$ and $P(k)$ will be consistent with each other. We also show that it is possible to extract the same amount of acoustic information by fitting over a finite range using either $\omega_{0}\left(r_{s}\right)$ or $P(k)$ derived from equal volume surveys.
\end{abstract}

Subject headings: distance scale — cosmological parameters — large-scale structure of universe cosmology: theory

\section{INTRODUCTION}

Baryon acoustic oscillations (BAOs) are relic imprints on the baryon distribution left behind by the interaction between radiation and matter in the primordial universe. The large amount of radiation pressure due to high temperatures in the early universe pushed matter apart as it congregated under the influence of gravity. Oscillating sound waves were set up in the primordial plasma due to these two competing effects (Peebles \& Yu 1970; Sunvaev \& Zeldovich 1970; Bond \& Efstathiou 1984; Holtzman 1989; $\mathrm{Hu}$ \& Sugivama 1996; $\mathrm{Hu}$ \& White 1996; Eisenstein \& Hu 1998). The distance traveled by these sound waves before recombination is about 150 comoving $\mathrm{Mpc}$ and is known as the acoustic scale. When the photons stream off, the baryons are deposited at these characteristic separations and hence we would expect excesses with this separation in the baryon distribution today. This characteristic scale makes for a very useful standard ruler (Eisenstein \& Hu 1998; Eisenstein et al. 1998; Eisenstein 2003; Blake \& Glazebrook 2003; Hu \& Haiman 2003; Linder 2003; Seo \& Eisenstein 2003; Matsubara 2004; Amendola et al. 2005).

As the universe evolves, primordial density perturbations begin to grow nonlinearly, especially on

\footnotetext{
${ }^{1}$ Steward Observatory, University of Arizona, 933 N. Cherry Ave., Tucson, AZ 85121; xxu@as.arizona.edu

${ }^{2}$ Departments of Physics and Astronomy, University of California, Berkeley, CA 94720

${ }^{3}$ Lawrence Berkeley National Laboratory, 1 Cyclotron Road, Berkeley, CA

${ }^{4}$ Department of Physics, Yale University, New Haven, CT 06511

${ }^{5}$ Center for Particle Astrophysics, Fermi National Accelerator Laboratory, P.O. Box 500, Batavia, IL 60510-5011
}

small scales (Jain \& Bertschinger 1994: Meiksin et al. 1999; Meiksin \& White 1999; Scoccimarro et al. 1999). This causes the acoustic scale to become slightly shifted from its predicted linear theory position (Seo \& Eisenstein 2005; Huff et al. 2007; $\mathrm{Ma}$ 2007; Angulo et al. 2008; Crocce \& Scoccimarro 2008; Sanchez et al. 2008; Seo et al. 2008; Smith et al. 2008; Padmanabhan \& White 2009). One must calibrate this shift before the acoustic scale can be used as a high precision standard ruler.

Previous calibrations and other analyses of the acoustic signature have been conducted using the traditional power spectrum and correlation function methods. Although analytically they are both perfectly adequate, the estimators used to derive them from simulations and observational data are subject to numerous difficulties.

The largest survey scales are always poorly constrained due to effects such as the integral constraint making it a challenge to estimate the correlation function $\xi(r)$ accurately at these scales. The integral constraint arises due to the fact that we do not know the cosmic number density of any population of mass tracers de Lapparent et al. 1988; Baumgart \& Frv 1991; Peacock \& Nicholson 1991; Hamilton 1993). Many techniques used to estimate $\xi(r)$ (Peebles 1973; Sharp 1979; Hewett 1982; Blanchard \& Alimini 1988; Landy \& Szalay 1993; Hamilton 1993) take the number density of tracers in the survey volume to be the true number density. This assumption effectively ignores all power at scales larger than the survey size while simultaneously increasing the correlation between scales smaller than the survey size which causes the off-diagonal covariance matrix terms to be larger than they would be otherwise. 
Limited survey volume and awkward survey boundaries are the major concerns when trying to estimate the power spectrum $P(k)$. Typically, the measured power spectrum is a convolution of the window function, the Fourier transform of the selection function of the survey, and the underlying true power spectrum (Feldman et al. 1994; Park et al. 1992; Baumgart \& Fry 1991; Peacock \& Nicholson 1991; Kaiser \& Peacock 1991). Therefore, these $P(k)$ estimators are biased. In the limit of infinite volume, the window function should be a delta function. However, real surveys have finite volume and hence the window function has a finite albeit very small width. This induces an artificial smoothing at small separations in $k$ when attempting to deconvolve the window function from the observed density field. Pair counting estimators, like those for $\xi(r)$, avoid this issue because the relative positions of all tracer particles and hence the true distribution is recorded.

Estimating $\xi(r)$ and $P(k)$ also requires the binning of data. When any binning process is invoked, one must carefully define any averaging used to derive the value in each bin. To reduce these errors, bin sizes are made smaller which increases the dimensionality of the covariance matrix, making it even more difficult to estimate. Also, survey boundaries need to be addressed with special care.

In the study of large scale structure, we are also interested in any anisotropies in the distribution of objects. These can result from the bulk motions of objects (Peebles 1980; Davis \& Peebles 1983; Kaiser 1987) as well as assumed models for the Hubble parameter $H(z)$ and the angular diameter distance $D_{A}(z)$ while calculating object separations along the line of sight (LOS) and transverse directions respectively. Since, at large scales we would expect the distribution to be isotropic, any anisotropy can be deconstructed into velocity field information which in turn provides us with constraints on $\Omega_{m}$, the derivative of the growth function $f=d \ln D / d \ln a$ and the anisotropic parameter $\beta=f / b$, where $b$ is the galaxy bias (Kaiser 1987; Hamilton 1998; Peacock et al. 2001; Hawkins et al. 2003; Tegmark et al. 2002; Zehavi et al. 2002; Song \& Percival 2008; Percival \& White 2009; White et al. 2009). After modeling these anisotropies, any residual anisotropies can be used to constrain $D_{A}(z) H(z)$ (Alcock \& Paczynski 1979). If our assumed $H(z)$ or $D_{A}(z)$ models are incorrect then the distribution of objects will still appear anisotropic after the bulk motion effects are removed. The magnitude of this residual anisotropy can be used to infer the true underlying cosmology (Padmanabhan \& White 2008; Okumura et al. 2008).

Anisotropic information can be extracted from the redshift-space correlation function and to a lesser extent from the redshift space power spectrum Szalay et al. 1998). If one imagines a wide angle survey, $P(k)$ estimators that rely on a Fourier transform from a Cartesian grid will suffer because an arbitrary wave vector $(\vec{k})$ will not necessarily be parallel to the LOS (Cole et al. 1994, 1995; Zaroubi \& Hoffman 1995; Szapudi 2004). This means that each $\vec{k}$ mode will contain information about both the LOS and transverse distributions. After averaging to obtain the spherically averaged power spectrum, any anistropies in the distribution of survey objects will have been erased. Instead of taking a spherical average, one can also analyze the full 3D $P(\vec{k})$ through Legendre decomposition into radial and angular components. However, an infinite sum is required and applicability is limited to the linear regime (Heavens \& Taylor 1995; Percival et al. 2004). Pair count estimators for $\xi(r)$, on the other hand, record the distribution of tracers accurately because they record each pair's angle to the LOS as well as their separation. This means that any anisotropies in the distribution of galaxies will become obvious. Statistics sensitive to anisotropic clustering are desirable as they offer us a means to probe the underlying cosmology.

It is also aesthetically pleasing, to localize the acoustic information into a single feature at the acoustic scale. This is true for $\xi(r)$ but not for $P(k)$, which has oscillatory acoustic features.

Although all of the above mentioned disadvantages of $\xi(r)$ and $P(k)$ are minor, it is still beneficial to derive a new statistic that does away with as many of the above setbacks as possible. $\omega_{\ell}\left(r_{s}\right)$ is an example of such an alternative.

The organization of this paper will be as follows. In 92 , we introduce $\omega_{\ell}\left(r_{s}\right)$, including its properties and computation. In \$3, we describe the simulations, halo occupation models and analysis methods we use to derive acoustic peak shifts through implementation of the monopole $\omega_{0}\left(r_{s}\right)$. We demonstrate the mutual consistency between the peak shifts measured from the same simulations using $\omega_{0}\left(r_{s}\right)$ and $P(k)$ in 4 . This is indicative of the agreement we expect between distance measures from $\omega_{0}\left(r_{s}\right)$ and $P(k)$. We also show that with a reasonable finite fitting range and our current choice of filter for computing $\omega_{\ell}\left(r_{s}\right)$, we can extract the same amount of acoustic information using either $\omega_{0}\left(r_{s}\right)$ or $P(k)$ from equal volume surveys. Finally, we state the main results of this paper in 95 . Details of the matter and galaxy model $P(k)$ results discussed in this paper can be found in the companion papers Seo et al. (2010) and Mehta et al. (in prep) respectively (hereafter referred to as S\&M collectively).

\section{THE $\omega_{\ell}\left(r_{s}\right)$ STATISTIC \\ 2.1. Equations and Properties}

We expand the angle dependence of the power spectrum and correlation function out as a series of Legendre polynomials in $\mu=\hat{r} \cdot \hat{z}=\cos (\theta)$, where $\theta$ is the LOS angle:

$$
\begin{aligned}
\xi(r, \mu) & \equiv \sum_{\ell} \xi_{\ell}(r) L_{\ell}(\mu) \\
\Delta^{2}(k, \mu) & \equiv \frac{k^{3} P(k, \mu)}{2 \pi^{2}}=\sum_{\ell} \Delta_{\ell}^{2}(k) L_{\ell}(\mu)
\end{aligned}
$$

so that

$$
\xi_{\ell}(r)=i^{\ell} \int \frac{d k}{k} \Delta_{\ell}^{2}(k) j_{\ell}(k r)
$$

where $j_{\ell}$ is the spherical Bessel function of order $\ell$ and $L_{\ell}$ is the Legendre polynomial of order $\ell$.

Imagine we have a filter, $W_{\ell}\left(r, \mu, r_{s}\right)=W_{\ell}\left(r, r_{s}\right) L_{\ell}(\mu)$, which we take to be compact and compensated $\left(\int r^{2} d r W_{\ell}\left(r, r_{s}\right)=0\right)$ with a characteristic scale $r_{s}$. We 
define our statistic as the redshift-space correlation function, $\xi_{s}(r, \mu)$, convolved with the filter as a function of filtering scale $r_{s}$.

$$
\begin{aligned}
\omega_{\ell}\left(r_{s}\right) & \equiv i^{\ell} \int d^{3} r \xi_{s}(r, \mu) W_{\ell}\left(r, r_{s}\right) L_{\ell}(\mu) \\
& =\frac{4 \pi i^{\ell}}{2 \ell+1} \int r^{2} d r \xi_{\ell}(r) W_{\ell}\left(r, r_{s}\right) \\
& =\int \frac{d k}{k} \Delta_{\ell}^{2}(k) \widetilde{W}_{\ell}\left(k, r_{s}\right)
\end{aligned}
$$

with

$$
\widetilde{W}_{\ell}\left(k, r_{s}\right) \equiv(-1)^{\ell} \frac{4 \pi}{2 \ell+1} \int r^{2} d r W_{\ell}\left(r, r_{s}\right) j_{\ell}(k r)
$$

where the $i^{\ell}$ has been inserted for later convenience. By making the filter compensated, we reduce the sensitivity to the poorly constrained power at large scales and the dependence on the uncertain mean density in the sample. The correlation function is defined such that $\xi(r, \mu)+1 \propto n^{-2}$ (Peebles 1980). Integrating the lefthand side of this equation against $W_{\ell}\left(r, r_{s}\right) L_{\ell}(\mu) d^{3} r$ results in $\omega_{\ell}\left(r_{s}\right)$ scaling directly with $n^{-2}$ following equation (41). The constant term integrates to 0 as the filter is compensated. Hence, any uncertainty in $n$ enters as a pure multiplicative offset in $\omega_{\ell}\left(r_{s}\right)$, which is less likely to overwhelm the acoustic signature at large scales. This in essence, eliminates sensitivity to the integral constraint, which is a small effect to begin with. This feature of the filter also makes the statistic measured in different subvolumes of a survey more independent. We expect that this will make internal error estimates from methods such as bootstrap or jackknife more robust (Padmanabhan et al. 2009).

Following Padmanabhan et al. (2007) we consider a low order, smooth compensated filter. For simplicity we assume $W_{\ell}$ is independent of $\ell$, though we could of course choose different weights for each multipole. In terms of $x \equiv\left(r / r_{s}\right)^{3}$, the filter

$$
W(x)=(2 x)^{2}(1-x)^{2}\left(\frac{1}{2}-x\right) \frac{1}{r_{s}^{3}}
$$

satisfies $W(0)=W^{\prime}(0)=W(1)=W^{\prime}(1)=0$ and $\int d x W(x)=0$. The suggested form in configuration space (top panel of Figure 11) has a broad hump peaking at $r \simeq 0.65 r_{s}$ that matches the width of the acoustic peak in $\xi(r)$ and a sharp (negative) spike at $0.9 r_{s}$ of width $\mathcal{O}(10 \%)$. This filter will smear a feature, such as the acoustic peak in $\xi(r)$, by very little which means that the acoustic information will be localized in $\omega_{\ell}\left(r_{s}\right)$, however, not as localized as in $\xi(r)$. Obviously, given sufficient signal-to-noise, measuring $\omega_{\ell}\left(r_{s}\right)$ for many $r_{s}$ values would allow resolution in $\xi(r)$ even below the intrinsic width of $W\left(r / r_{s}\right)$ (see $\left.\$ 2.3\right)$.

6 For example, we could make the $k$-weight for $\ell=0$ and $\ell=2$ equal. Doing so facilitates the computation of $Q(r)=$ $\frac{\xi_{2}(r)}{3 / r^{2} \int_{0}^{r} \xi\left(r^{\prime}\right) r^{\prime 2} d r^{\prime}-\xi_{0}(r)}=\frac{4 / 3 \beta+4 / 7 \beta^{2}}{1+2 / 3 \beta+(1 / 5) \beta^{2}}$ (Hamilton 1992), when transformed to $r_{s}$ space, since it involves both the monopole and the quadrupole. This ratio is useful for estimating the anisotropic parameter $\beta$, however, it has limited applicability outside linear theory.
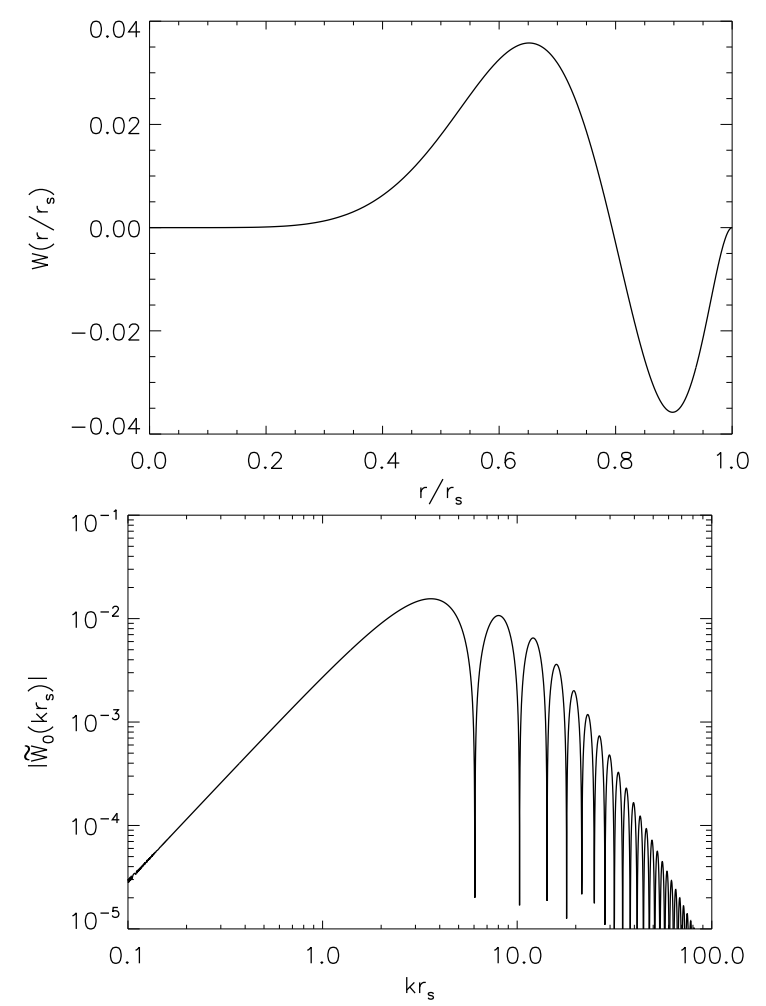

FIG. 1.- (top) The filter $W_{\ell}\left(r / r_{s}\right)$ in configuration space. There is a broad hump matching the width of the acoustic peak in $\xi(r)$ which peaks at $r \simeq 0.65 r_{s}$ and a sharp (negative) spike at $0.9 r_{s}$ with a width on the order of $10 \%$. A filter of this shape will smear a feature, such as the acoustic peak in $\xi(r)$, by only a small amount which means that the acoustic information will be well localized in $\omega_{\ell}\left(r_{s}\right)$. Its compensated nature implies that $\omega_{\ell}\left(r_{s}\right)$ is not sensitive to the integral constraint. (bottom) The filter $\widetilde{W}_{\ell}\left(k r_{s}\right)$ for $\ell=0$. The insensitivity of this filter to large scales is reflected in the fact that it is singly compensated and $\widetilde{W}_{\ell} \sim k^{2}$ as $k \rightarrow 0$. At small scales $\widetilde{W}_{\ell}\left(k r_{s}\right) \rightarrow \cos \left(k r_{s}\right) /\left(k r_{s}\right)^{4}$. This is a much more rapid drop-off than observed in the kernel for $\xi(r)$, which scales as $(k r)^{-1}$. These properties of the filter imply that $\omega_{\ell}\left(r_{s}\right)$ only probes a narrow range of scales in Fourier space, and that it is insensitive to large scale fluctuations or poorly constrained smallscale structure.

With this choice of $W_{\ell}$ the window function $\widetilde{W}_{\ell}$ can be computed analytically (see Appendix for numerical details) or numerically via fast Hankel transforms. We show $\widetilde{W}_{\ell}\left(k r_{s}\right)$ for $\ell=0$, in the bottom panel of Figure 1. Since the filter is singly compensated, $\widetilde{W}_{\ell} \sim k^{2}$ as $k \rightarrow 0$, reflecting insensitivity to large scales. At small scales $\widetilde{W}_{\ell}\left(k r_{s}\right) \rightarrow \cos \left(k r_{s}\right) /\left(k r_{s}\right)^{4}$, a much more rapid convergence than evinced by the kernel for $\xi(r)$, which scales as $(k r)^{-1}$. Thus $\omega_{\ell}$ probes a narrow range of scales in Fourier space and is insensitive to fluctuations on large scales or poorly measured or modeled small-scale structure. One can choose the range of $k$ to be sampled by appropriate choice of $r_{s}$ : more information from high $k$ modes can be included by using smaller $r_{s}$.

As an example, the linear theory monopole statistic $\omega_{0}\left(r_{s}\right)$ is plotted in Figure 2 Plotting $r_{s}^{2} \omega_{0}$ versus $r_{s}$ gives a convenient vertical range. The acoustic information is mostly localized into a single dip around the acoustic scale (see $\longdiv { 4 . 2 . 1 }$ ).

\subsection{Computation}




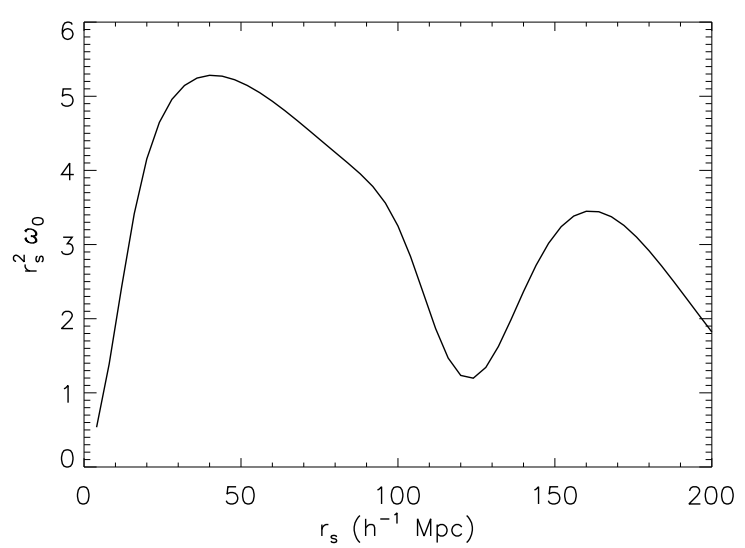

FIG. 2.- Linear theory monopole statistic $\omega_{0}\left(r_{s}\right)$. The acoustic information can be seen around the acoustic scale, mainly localized into a single dip feature.

It is possible to adapt $\omega_{\ell}\left(r_{s}\right)$ into a sum over unbinned pair counts for any sample of mass tracers following the methods described in Padmanabhan et al. (2007); there is no need to first compute $\xi(r, \mu)$ via binning of data. Pair counting allows us to record each galaxy's angle to the LOS $(\mu)$ accurately. Hence, like $\xi(r), \omega_{\ell}\left(r_{s}\right)$ is sensitive to any anisotropies of the tracer distribution in clusters (discussed in \$1).

The redshift-space correlation function can be estimated as

$$
\xi_{s}(r, \mu)=\frac{D D(r, \mu)}{R R(r, \mu)}-1,
$$

where $D D(r, \mu)$ is the number of data tracer pairs separated by $r$ and have LOS angle corresonding to $\mu$. $R R(r, \mu)$ is the analogue for randomly distributed points, normalized to the data counts by a factor of $N_{D}^{2} / N_{R}^{2}$. Here, $N_{D}$ and $N_{R}$ are the total number of data and random points respectively. When analyzing observational data, the number of random points needs to be much larger than the number of data points to keep the shotnoise in $R R$ smaller than that in $D D$, especially at small $r$. For simulation data, however, it is not necessary to use a very large number of random points to compute $R R$ smoothly at small scales (elicited below).

Equation (9) implies that Equation (4) can be rewritten as

$$
\omega_{\ell}\left(r_{s}\right)=i^{\ell} \int d^{3} r W_{\ell}(r) L_{\ell}(\mu) \frac{D D(r, \mu)}{R R(r, \mu)} .
$$

The -1 integrates to 0 due to the compensated nature of the filter.

The $R R$ piece is purely geometrical and is dependent only on the survey geometry (encoded in $\Phi(r, \mu)$ ) and the number of random points. Hence we can write $R R$ as

$$
R R(r, \mu)=2 \pi n_{D} N_{D} r^{2} \Phi(r, \mu) d r d \mu
$$

where $n_{D}$ is the number density of data points which is easily calculable for surveys with well defined boundaries. The above equation defines $\Phi(r, \mu)$ to be any mismatch between infinite sized surveys/simulations and finite sized ones due to the presense of boundaries. The $n_{D} N_{D}$ factor is due to the normalization of the RR counts as mentioned above. For observations, $\Phi(r, \mu)$ can be computed via binning methods and then fit using a smooth function $\hat{\Phi}(r, \mu)$. Note that the binning mentioned here is only required in the computation of the $R R$ counts; there is no need to bin the data. In the case of simulations in a periodic box, $\hat{\Phi}(r, \mu)$ is constant as the volume is effectively infinite.

With these points in mind, we can now pick arbitrarily small bins when computing the $D D$ counts since $R R$ has been approximated by a smooth function and hence does not suffer from shot-noise induced through pair-counting. As is such, we may employ a binning scheme in which there is either zero or one $D D$ pair per bin. This step reduces the integral in Equation (10) to a sum over $D D$ pairs as in

$$
\omega_{\ell}\left(r_{s}\right)=i^{\ell} \sum_{i \in D D} \frac{W_{\ell}\left(r_{i}\right) L\left(\mu_{i}\right)}{n_{D} N_{D} V \hat{\Phi}\left(r_{i}, \mu_{i}\right)} .
$$

Since the estimator can be written as a summation, there is no longer a need to bin data at all.

\subsection{Covariance Matrix}

Since $\omega_{\ell}$ does not require the binning of data, we can in principle estimate it at as many $r_{s}$ values as we wish without affecting the signal in the adjacent values: there is no bin which is made smaller. However adjacent points become increasingly correlated as the $r_{s}$ spacing decreases, compromising the usefulness of very fine sampling.

In the Gaussian limit, the covariance matrix is

$$
\begin{gathered}
\operatorname{Cov}\left[\omega_{\ell}\left(r_{s}\right), \omega_{\ell^{\prime}}\left(r_{s}^{\prime}\right)\right]=\frac{2(2 \ell+1)\left(2 \ell^{\prime}+1\right)}{V} \\
\times \int \frac{k^{2} d k}{2 \pi^{2}} \widetilde{W}_{\ell}\left(k r_{s}\right) \widetilde{W}_{\ell^{\prime}}\left(k r_{s}^{\prime}\right) \mathcal{I}_{\ell \ell^{\prime}}(k)
\end{gathered}
$$

with

$$
\mathcal{I}_{\ell \ell^{\prime}}=\frac{1}{2} \int d \mu L_{\ell}(\mu) L_{\ell^{\prime}}(\mu)\left[\sum_{L} P_{L}(k) L_{L}(\mu)+\aleph\right]^{2}
$$

where $\sum P_{L}(k) L_{L}(\mu)$ is the legendre decomposition of the full 3D power spectrum $P(\vec{k})$ and $\aleph$ is shot-noise. Assuming Poisson shot-noise, $\aleph=\bar{n}^{-1}$, where $\bar{n}$ is the number density of the mass tracer.

\subsection{Summary of Key Features}

We conclude this section with a summary of the key features and advantages of $\omega_{\ell}\left(r_{s}\right)$ over $\xi(r)$ and $P(k)$ estimators.

1. $\omega_{\ell}\left(r_{s}\right)$ has a compensated filter that reduces sensitivity to poorly constrained large scale power and hence the integral constraint. $\xi(r)$, on the other hand, experiences these problems. The compensated filter also makes $\omega_{\ell}\left(r_{s}\right)$ measured in different subvolumes of the survey more independent which is important for attaining robust error estimates from methods such as bootstrap and jackknife.

2. The filter is approximately compact in both configuration and Fourier space. The smoothness in configuration space leads to the steep drop-off at high $k$ in Fourier space. This effectively minimizes the impact of large $k$ or small scale power which is 
not well constrained in large cosmological surveys. The filter is localized in configuration space which means that, unlike in $P(k)$, the acoustic information is localized in $\omega_{\ell}\left(r_{s}\right)$. However, it is not as localized as in $\xi(r)$ (see \$4.2.1).

3. Like $\xi(r), \omega_{\ell}\left(r_{s}\right)$ can be easily adapted into a pair count statistic, so the relative positions of tracers and each pair's angle from the LOS is accurately recorded. Hence, it estimates the underlying galaxy distribution without the need to deconvolve a window function (as in the case of $P(k)$ ) and is sensitive to any anisotropies in the clustering of tracers. The anisotropies can be used to determine the underlying cosmology. $P(k)$ estimators do not typically allow this type of analysis.

4. There is no need to bin data when computing $\omega_{\ell}\left(r_{s}\right)$, unlike when estimating $\xi(r)$ and $P(k)$.

While the setbacks of traditional $P(k)$ and $\xi(r)$ estimators are minor, it is beneficial to have a statistic like $\omega_{\ell}\left(r_{s}\right)$ which combines many of the advantages of both.

\section{SIMULATIONS AND ANALYSIS METHODS}

A major goal in developing this new statistic is to use it for better measuring and calibrating the acoustic scale. The monopole statistic $\omega_{0}\left(r_{s}\right)$ is especially useful in this regard as it is a direct map from the traditional 2-point correlation function and power spectrum. Therefore, any results obtained from $\mathrm{BAO}$ analysis via these three statistics is readily comparable. As with the quadrupole of the power spectrum $P_{2}(k)$ (Padmanabhan \& White 2008), anisotropic BAO analysis can be performed using the quadrupole $\omega_{2}\left(r_{s}\right)$. However, as the main goal of this paper is to give a broad introduction to the $\omega_{\ell}\left(r_{s}\right)$ statistic and a simple demonstration of its application, we defer detailed discussion of $\omega_{2}\left(r_{s}\right)$ and anistropic BAO to a future paper.

In this section, we implement $\omega_{0}\left(r_{s}\right)$ through the use of pure dark matter N-body simulations. It is possible to model a variety of different galaxy populations and biases through application of appropriate HODs to the halos found in the simulations. This is important in demonstrating the robustness of the $\omega_{\ell}\left(r_{s}\right)$ statistic over a diverse set of galaxy populations. We compute $\omega_{0}\left(r_{s}\right)$ for dark matter and the mock galaxy populations created via the HODs, and demonstrate how it can be used to measure the shift in the acoustic peak. This quantity is important in constraining the precise size of the acoustic scale, which may be slightly different from that predicted by linear theory due to nonlinear structure growth. In order to quote the shift with accurate errors, we use a resampling technique described in 3.4 which gives us a large number of shifts from which to calculate a mean and a standard error of that mean.

\subsection{Simulations}

Our simulations were performed using a code developed by Metchnik and Pinto which employs a new method (Metchnik \& Pinto 2010) to compute N-body forces under periodic boundary conditions. Rather than resorting to the Fourier methods of PM schemes or using Ewald sums to represent periodicity, this new method represents periodicity directly, expressing the force on a particle as due to the rest of the simulation volume and an infinite sum over its periodic images.

This is made more efficient by partitioning the computational domain into a three-dimensional grid. The acceleration on particles within a grid cell is divided into two parts: a near field and a far field. The near field is that due to the other particles in the cell and to particles in the adjacent 26 cells. In these calculations, the nearfield acceleration was computed using the direct, $O\left(N^{2}\right)$ method, with Plummer softening.

The far field acceleration on particles in the cell due to each more distant cell on the grid is represented as a Taylor series expansion based on the multipole moments in the distant cell. The contribution from all periodic images of the distant cell is included by recognizing that the multipole moments in a cell are identical to those in all of its images. Thus, the sum over images depends only on the (fixed) geometry of the grid and need be performed only once. The (relatively) small set of values which results provides a simple and rapidly-evaluated relation between the multipole moments in distant grid cells and the Taylor coefficients of the expansion for the acceleration in a given cell, all under periodic boundary conditions. The acceleration due to all of the periodic images of the cell and its 26 neighbors is included in a similar manner.

The calculations described here used order-16 expansions, providing an overall force accuracy per particle which agrees with Ewald summation to better than six decimal digits. Overall, the method is significantly faster and requires significantly less memory than other methods for computing accelerations in large, periodic N-body problems, while providing accelerations to machine precision for all particles. Time integration was performed using a standard kick-drift-kick algorithm, using independent time steps for each particle.

We derive our results in redshift-space at $z=1$ from a set of 44 simulations with $1024^{3}$ dark matter particles in each. The simulations were performed in $1 h^{-1}$ Gpc periodic boxes with the WMAP $5+\mathrm{SN}+\mathrm{BAO}$ best-fit cosmological parameters: $\Omega_{m}=0.279, \Omega_{\Lambda}=0.721, h=0.701$, $\Omega_{b}=0.0462, n_{s}=0.96$ and $\sigma_{8}=0.817$ (Komatsu et al. 2009) which implies a particle mass of $7.2 \times 10^{10} h^{-1} M_{\odot}$. The initial conditions are generated via the second-order Lagrangian perturbation theory code of Sirko (2005) at $z=50$ with no extra power for the box scale.

\subsection{Halo Occupation Distributions}

We use a simple friends-of-friends (Davis et al. 1985) method with a linking length equal to 0.16 of the interparticle spacing to identify the collapsed dark matter halos in our simulations. We then populate these halos with galaxies by applying simple HODs based on the form

$$
\left\langle N_{g}(M)\right\rangle=\left[1+\left(M / M_{\text {sat }}\right)^{\gamma}\right] \exp \left(-M_{\text {cen }} / M\right)
$$

where $M$ is the halo mass, $M_{\text {cen }}$ is the minimum mass for a halo to contain a central galaxy, $M_{\text {sat }}$ is the minimum mass for a halo to contain at least one satellite, and $\gamma$ is an exponential parameter that we set to 1 (Guzik \& Seliak 2002; Berlind et al. 2003; Kravtsov et al. 2004; Zheng et al. 2005). We assign a 
central galaxy to a halo if $M>M_{\text {cen }}$, this is a good approximation to $\left\langle N_{\text {cen }}(M)\right\rangle=\exp \left(-M_{\text {cen }} / M\right)$. The central galaxy is taken to be at the halo's center of mass and assigned the center of mass velocity. If a halo is assigned a central galaxy, then the number of satellite galaxies is determined by generation of a random integer based on a Poisson distribution with mean equal to $\left\langle N_{\text {sat }}(M)\right\rangle=\left(M / M_{\text {sat }}\right)^{\gamma}$. We then randomly pick a corresponding number of halo particles and assign their positions and velocities to the satellites.

In order to compare the peak shifts derived using $\omega_{0}\left(r_{s}\right)$ and $P(k)$ in a range of models, we apply three different HODs to our simulations. The properties of these HODs are described in Table 1 and obtained by adjusting the values of $M_{\text {cen }}$ and $M_{\text {sat }}$. We also list the properties of a dark matter (DM) only case that is merely a $0.4 \%$ subsample of the particles in each simulation. We compute $\omega_{0}\left(r_{s}\right)$ in redshift-space via the pair counting method detailed in 2.2 within the range $5 h^{-1} \mathrm{Mpc} \leq r_{s} \leq 200 h^{-1} \mathrm{Mpc}$ using $5 h^{-1} \mathrm{Mpc}$ spacing. We also compute $\sigma_{8}$, the RMS mass fluctuation within a $8 h^{-1} \mathrm{Mpc}$ radius, using a similar pair counting method derived from the configuration space equation for a general radius $\mathrm{R}$ (Zehavi et al. (2005))

$$
\sigma_{R}^{2}=\int_{0}^{2 R} \frac{1}{R^{3}}\left[3-\frac{9}{4} \frac{r}{R}+\frac{3}{16}\left(\frac{r}{R}\right)^{3}\right] r^{2} \xi(r) d r .
$$

\subsection{Fitting $\omega_{0}\left(r_{s}\right)$ to Measure the Peak Shift}

We fit the redshift-space $\omega_{0}\left(r_{s}\right)$ using the form

$$
P_{\text {sim }}(k)=B(k) P_{m}(k / \alpha)+A(k)
$$

where

$$
B(k)=\frac{\left(b_{1}^{2}+b_{2}^{2} k+b_{3}^{2} k^{2}\right)}{1+r_{\text {scale }} k}
$$

and $A(k)$ transforms into $A\left(r_{s}\right)=a_{1} r_{s}^{-9}$ in $r_{s}$ space. Such a form for $A(k)$ is motivated by the fact that we want to marginalize over the shape of the correlation function at small scales (i.e., the contribution of the 1-halo term). Expanding Equation (8), we see that $W(x)=\left(-4 x^{5}+2 x^{4}-8 x^{3}+2 x^{2}\right) \frac{1}{r_{s}^{3}}$. This implies that the highest order term in $r_{s}$ is $r_{s}^{-9}$. At small scales, this is the term that will dominate in the transformation from $\xi(r)$ to $\omega_{0}\left(r_{s}\right)$ as defined by Equation (44).

$P_{m}(k)$ is the template power spectrum we use for our fitting. To account for the degradation of the acoustic peak through nonlinear evolution and redshift distortions, the template model $P_{m}(k)$ is obtained from the linear power spectrum $P_{\text {lin }}(k)$ at $z=1$ by the modification

$$
P_{m}(k)=\left[P_{\text {lin }}(k)-P_{\text {smooth }}(k)\right] \exp \left(-k^{2} \Sigma_{\text {nl }}^{2} / 2\right)+P_{\text {smooth }}(k)
$$

where $P_{\text {smooth }}$ is the dewiggled power spectrum described in Eisenstein \& Hu (1998) and $\Sigma_{n l}$ is a nonlinear parameter used to degrade the peak (Eisenstein et al. 2005; Tegmark et al. 2006; Crocce \& Scoccimarro 2006; Eisenstein et al. 2007; Crocce \& Scoccimarro 2008; Matsubara 2008). To allow maximum flexibility in our marginalization, we marginalize over $\Sigma_{\mathrm{nl}}$ and the other nonlinear nuisance parameter in equation (18), $r_{\text {scale }}$.

Our scale dilation parameter $\alpha$ represents the shift in the acoustic peak. Under this formalism, $\alpha>1$ indicates a shift towards smaller scales and $\alpha<1$ indicates a shift towards larger scales. Physically, $\alpha$ is the ratio between the linear theory acoustic scale $(150 \mathrm{Mpc})$ to the measured acoustic scale. Since all the terms in the fitting function are additive, the basis functions $P_{m}(k / \alpha) /(1+$ $\left.r_{\text {scale }} k\right), k P_{m}(k / \alpha) /\left(1+r_{\text {scale }} k\right), k^{2} P_{m}(k / \alpha) /\left(1+r_{\text {scale }} k\right)$, and $r_{s}^{-9}$ for a fixed $\alpha, \Sigma_{\mathrm{nl}}$ and $r_{\text {scale }}$ can be easily mapped into $r_{s}$ space (if necessary) using equation (6). A leastsquares fit using the mapped basis functions is then performed against $\omega_{0}\left(r_{s}\right)$ from the simulations to obtain values for the linear nuisance parameters $b_{1}, b_{2}, b_{3}$ and $a_{1}$. As we are interested in the acoustic feature, we use a fitting range of $30 \leq r_{s} \leq 200 h^{-1} \mathrm{Mpc}$. For an $r_{s}$ spacing of $5 h^{-1} \mathrm{Mpc}$, this implies 28 degrees of freedom in the fit, where the number of degrees of freedom is defined as the difference between the number of data points being fit and the number of parameters in the fitting form.

We assume that the errors on $\omega_{0}\left(r_{s}\right)$ can be well approximated by the covariance matrix $C$ assuming Poisson shot-noise (see 3.4 ) with the addition of nonlinear shotnoise (see equation (20) and surrounding text). We also assume that the monopole $(\ell=0)$ dominates $P(\vec{k})$ so that all higher order contributions to the power spectrum are effectively zero. This amounts to computing $C$ using $P(\vec{k})=P_{m}(k)$ as the input power spectrum, where we take a fixed $\Sigma_{\mathrm{nl}}=7.0 h^{-1} \mathrm{Mpc}$ at $z=1$ in redshift-space following Seo et al. (2008). We normalize this power spectrum to the amplitude of the redshift-space power spectrum through multiplication by the bias squared defined initially as $b^{2}=\left(\sigma_{8, \text { case }} / \sigma_{8, \text { matter }}\right)^{2}$. The values for $\sigma_{8 \text {,case }}$ are given in Table 2 and $\sigma_{8 \text {,matter }}=0.506$ in real space at $z=1$ in linear theory. We want to ensure that the input power spectrum to the covariance matrix calculation is as close as possible to the simulation data so that the covariance matrix is a reliable estimate of the errors. To do this, we marginalize over the average $\omega_{0}\left(r_{s}\right)$ of the 44 simulations for each case to obtain a value for the leading order term that scales $P_{m}(k): b_{1}^{2}$ in the fitting form of equation (18). We then iterate this marginalization and scale $b^{2}$ by the values of $b_{1}^{2}$ obtained until the output $b_{1}^{2}$ from this iterative fitting is close to 1. We expect that scaling $P_{m}(k)$ by this final value of $b^{2}$ will approximate the simulation data well and hence be valid input to the covariance matrix calculation for the resampling techniques described in 3.4

The shot-noise we enter into the calculation of $C$ includes a nonlinear component (quoted in Table 1) in addition to the Poisson shot-noise $\bar{n}^{-1}$ as described in Equation (13). This additional shot-noise is a result of nonlinear structure formation on small scales. We estimate this nonlinear shot-noise as

$$
\aleph_{\text {nonlin }}=\int_{0}^{r_{\text {nonlin }}} 4 \pi r^{2}\left[\xi(r)-\xi_{\text {lin }}(r)\right] d r
$$

where $\xi(r)$ is the correlation function averaged over the 44 simulations for each HOD, $\xi_{\text {lin }}(r)$ is the linear correlation function at $z=1$ and $r_{\text {nonlin }}$ is the scale above which nonlinear effects become unimportant. We take $r_{\text {nonlin }}$ to be $10 h^{-1} \mathrm{Mpc}$. The resulting $\aleph_{\text {nonlin }}$ is a rough estimate of the excess small scale correlation due to nonlinear evolution. Since it makes little difference whether all of this extra shot-noise comes in at zero separation in 


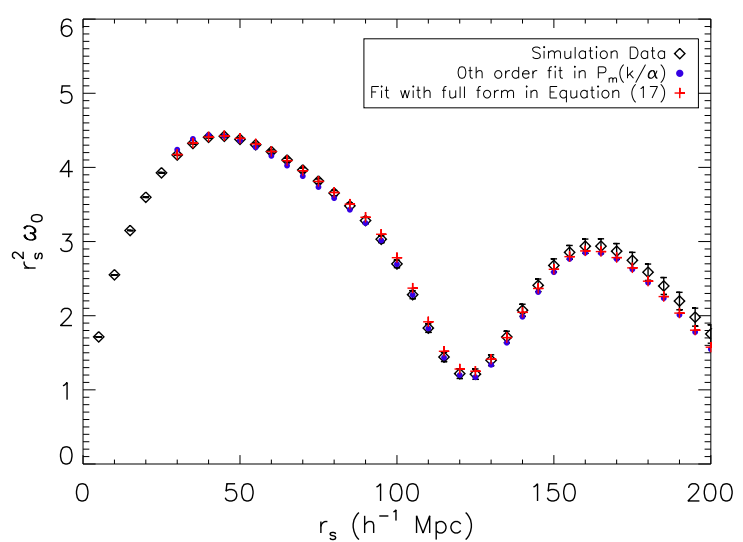

FIG. 3.- $\omega_{0}\left(r_{s}\right)$ averaged over all 44 simulations for HOD1 (black diamonds). Overplotted are a fit obtained through the form in Equation (17) (red crosses) and a 0th order fit in $P_{m}(k / \alpha)$ (purple dots), both over a range of $30 \leq r_{s} \leq 200 h^{-1} \mathrm{Mpc}$. One can see that the $0 t h$ order fit already appears quite good with $\chi^{2}=2.20$ per dof. However, by introducing additional nuisance parameters, the quality of the fit over the specified range improves further to $\chi^{2}=0.94$ per dof.

$r$ or through the extended effects of the one-halo term, which is only important at small $r$, we assume the excess correlation to be a spike at $r=0$ for convenience. When transformed into $k$ space, this gives a constant and becomes extra white noise that we add on to every mode equally, in addition to the Poisson shot-noise. For the subsampled DM case, the linear and the measured correlation functions were sufficiently similar at $1-10 h^{-1} \mathrm{Mpc}$ to warrant taking $\aleph_{\text {nonlin }}=0$ for this case. Alternatively, one can also account for nonlinear shot-noise by computing the covariance matrix using the nonlinear power spectrum, but this is more computationally challenging.

The $\chi^{2}$ likelihood indicator corresponding to the bestfit linear nuisance parameters for fixed $\alpha, \Sigma_{\mathrm{nl}}$ and $r_{\text {scale }}$ is then

$$
\chi^{2}=\left(\vec{\omega}_{0}-\vec{m}\right)^{T} C^{-1}\left(\vec{\omega}_{0}-\vec{m}\right)
$$

where $\vec{\omega}_{0}$ is $\omega_{0}\left(r_{s}\right)$ measured from the simulations, $\vec{m}$ is the best-fit model and $C^{-1}$ is the inverse of the covariance matrix. We compute the best-fit values of $\alpha, \Sigma_{\mathrm{nl}}$ and $r_{\text {scale }}$ by minimizing $\chi^{2}$ of the fits for the DM case and for each HOD using a generalized reduced gradient method from IDL. We quote the bias for each case as $\sigma_{8, \text { case }} / \sigma_{8, \text { matter }}$ multiplied by the additional scaling factors of $b_{1}$ described above.

We plot $\omega_{0}\left(r_{s}\right)$ averaged over all 44 simulations for HOD1 in Figure 3. Overplotted are the marginalization obtained through the form in Equation (17) and a 0th order fit obtained by just a rescaling of $P_{m}(k / \alpha)$, i.e. $b^{2} P_{m}(k / \alpha)$ where $b$ is the only fit parameter. Although the 0th order fit already appears quite good, one can see that introducing additional nuisance parameters improves the quality of the fit even more over the fitting range. The $\chi^{2}$ per degree of freedom (dof) improves from 2.20 to 0.94 .

Using this fitting technique, we derive values of bias, $\alpha$ and associated errors for each of our three HODs and our DM case via the resampling methods described in the following section.

\subsection{Resampling Methods}

We use two different methods to measure the mean peak shift $\alpha$ and the scatter in the mean $\sigma_{\alpha}$ for each case in Table 1. The first is a modified jackknife technique in which we randomly select $\mathrm{M}$ out of $\mathrm{N}$ simulations at a time without replacement, average their $\omega_{0}\left(r_{s}\right)$ and fit this average. We repeat this 1000 times and extract an average $\alpha$ and a scatter in $\alpha$. This scatter needs to be rescaled by an additional factor of $f=\sqrt{M} / \sqrt{N-M}$ in order to reflect the scatter asscociated with the mean of $\alpha$ for $\mathrm{N}$ simulations. For our simulations we have $N=44$ and take $M=22$. With this choice of $M, f=1$ and so the scatter in $\alpha$ reflects the error in the mean of $\alpha$. This method is useful in that it provides us with a large set of $\alpha$ 's from which we can accurately derive a mean $\alpha$ and $\sigma_{\alpha}$.

The fit results for the subsampled DM case as well as for each HOD model are quoted in Table 2. The average values of $\Sigma_{\mathrm{nl}}$ and $r_{\text {scale }}$ are also included for completeness, however the focus of this paper is on $\alpha$. The values of $\alpha$ we obtain are $1 \sigma$ consistent with those derived using the perturbation theory results of Padmanabhan \& White (2009). The somewhat low value of $\chi^{2}$ per dof for HOD3 suggests that we are overestimating the amount of nonlinear shot-noise. We are also approaching the shot-noise limited regime for HOD3 as evidenced by the fact $\bar{n} P_{0.2} \approx 1.6$. We note here that we used $\chi^{2}$ only to find the best-fit $\alpha$ for each HOD, not to generate the errors. Hence, the fact that our reduced $\chi^{2}$ values are slightly deviant from unity does not hinder the error estimation.

The second method we use is jackknife resampling. The results obtained using this method are in good agreement with the first method. This indicates that the error estimates obtained using our first method are robust in comparison to more traditional methods.

By using these resampling techniques, any nonGaussian effects not accounted for by assuming a Gaussian covariance matrix while fitting (as in $\$ 3.3$ ) will be reflected in $\sigma_{\alpha}$.

\section{COMPARISON TO THE POWER SPECTRUM}

\subsection{Comparison from Simulations}

An important step in implementing this new statistic is to show that it produces consistent results when compared to established methods and can therefore be an effective calibrator of the acoustic scale. We do this by comparing the peak shifts measured from the same set of simulations via the new $\omega_{0}\left(r_{s}\right)$ statistic and the traditional $P(k)$ method (see S\&M for details).

As we use the same 1000 random sets of $M$ simulations as S\&M, there should be a 1:1 correspondance between the $\alpha$ 's derived from $\omega_{0}\left(r_{s}\right)$ and $P(k)$ for the DM case and for each HOD. It should be noted that S\&M use different $P(k)$ fitting forms from the one detailed in 3.3 . They employ two fitting forms, both of which can also be described by equation (17). The first form has $B(k)$ as a $2^{\text {nd }}$ order polynomial and $A(k)$ as a $7^{\text {th }}$ order polynomial. The second form uses Pade approximants for $B(k)$, i.e., $B(k)=b_{0}\left(1+c_{1} k+c_{3} k^{2}+c_{5} k^{3}\right) /\left(1+c_{2} k+c_{4} k^{2}\right)$ and a $2^{\text {nd }}$ order polynomial for $A(k)$. We have chosen a different form in this work to induce better convergence of the integral from Fourier space to $r_{s}$ space while transforming the basis functions. We compare the $\alpha$ 's measured from 

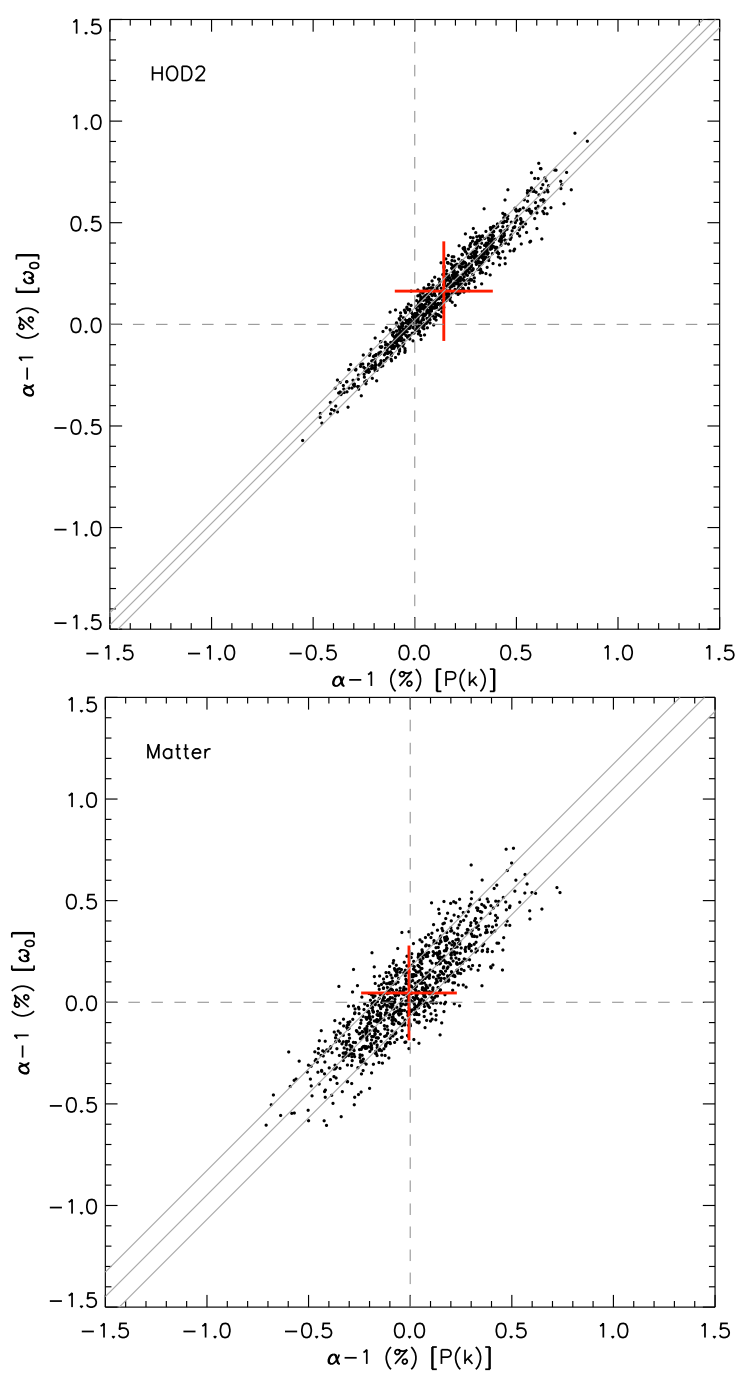

FIG. 4. $-\alpha$ from $\omega_{0}\left(r_{s}\right)$ versus $\alpha$ from $P(k)$ for HOD2 (top) and DM (bottom). The data points are from a resampling technique in which we randomly pick $M=22$ simulations out of $N=44$ total and fit the averaged $\omega_{0}\left(r_{s}\right)$ from these $M$ simulations. We repeat this 1000 times and hence obtain 1000 values of $\alpha$. The scatter on $\alpha$ needs to be rescaled by $\sqrt{M} / \sqrt{N-M}$ to reflect the true scatter on the mean. For our choice of $M$, this scaling factor is equal to 1 . Hence the scatter in the plot truly reflects the scatter on the mean of $\alpha$. The red cross marks the mean $\alpha$ values with their associated errors. The central grey line has unity slope and passes through the mean. The two outer grey lines delineate the $1 \sigma$ boundaries associated with $\Delta \alpha_{\omega P}$. As the data points lie largely in between the $1 \sigma$ lines with a slope similar to unity for both HOD2 and the DM case (see Table 3), we conclude that the two $\alpha$ sets are consistent with each other. The same correlation is observed for HOD1. HOD3 shows $1.6 \sigma$ agreement between $\omega_{0}\left(r_{s}\right)$ and $P(k)$. This slightly larger discrepancy may be due to the fact that shotnoise is becoming significant in this low number density case. Also, shot-noise may affect $P(k)$ and $\omega_{0}\left(r_{s}\right)$ differently or the nuisance parameters may not be fully handling the scale-dependence of a high-bias HOD such as HOD3. The large scatter in the DM case is likely due to the subsampling of matter in the computation of $\omega_{0}\left(r_{s}\right)$ but not in $P(k)$. The overall agreement between the $\omega_{0}\left(r_{s}\right)$ and $P(k)$ results imply that distance measures will be consistent between the two.

$\omega_{0}\left(r_{s}\right)$ against those measured from $P(k)$ by Seo et al. (2010) (DM) and Mehta et al. (in prep) (HODs) using the first form. It should also be noted here that the $P(k)$ results obtained for the DM case by Seo et al. (2010) utilize the full DM sample whereas we have subsampled to reduce computation time in this work. The methodology used to derive the $P(k)$ results are described in detail in Seo et al. (2010).

Figure 4 shows $\alpha$ from $\omega_{0}\left(r_{s}\right)$ versus $\alpha$ from $P(k)$ for the 1000 fit iterations performed on HOD2 (top) and DM (bottom). The red cross indicates the mean $\alpha$ values with their associated errors. The central grey line is a line with slope unity that passes through the mean. The two outer grey lines indicate the $1 \sigma$ boundaries associated with $\Delta \alpha_{\omega P}=\alpha_{\omega}-\alpha_{P}$. The mean difference between $\alpha_{\omega}$ and $\alpha_{P}$ along with the standard deviation is quoted in Table 3 under $\left\langle\Delta \alpha_{\omega P}\right\rangle$ for the DM case as well as each HOD case. The plots indicate that the correlation between $\alpha_{\omega}$ and $\alpha_{P}$ is $1 \sigma$ consistent with a line of slope unity that has a y-intercept of 0 for both HOD2 and the DM case. This implies that the two $\alpha$ sets are consistent with each other. The same holds for HOD1 as evidenced through the table, but HOD3 is slightly more deviant with $1.6 \sigma$ agreement between $\omega_{0}\left(r_{s}\right)$ and $P(k)$. The larger discrepancy between the HOD3 results may be due to the fact that shot-noise is becoming significant in this case (as shown in \$3.4). It could also be that shotnoise affects $P(k)$ and $\omega_{0}\left(r_{s}\right)$ differently or the nuisance parameters are not fully handling the scale-dependence of a high-bias HOD such as HOD3. The large scatter in the DM case is likely due to the fact that we have subsampled the matter in our computation of $\omega_{0}\left(r_{s}\right)$ but not in $P(k)$. The $\alpha$ 's from most of the cases are $1 \sigma$ consistent between $\omega_{0}\left(r_{s}\right)$ and $P(k)$, indicating that distance measures will be consistent between the two statistics. This also indicates that any systematics introduced by using the different fitting forms for $P(k)$ and $\omega_{0}\left(r_{s}\right)$ are minor. Hence we conclude that $\omega_{0}\left(r_{s}\right)$ is a well-tuned statistic for analysis of BAOs.

\subsection{Theory Constraints on $\sigma_{\alpha}$}

As we wish to promote $\omega_{0}\left(r_{s}\right)$ as an alternative method for analyzing the BAO, it is necessary to show how much acoustic information can be extracted from $\omega_{0}\left(r_{s}\right)$ relative to $P(k)$ and $\xi(r)$ for surveys of the same size. If our fitting ranges were infinite, then by the definitions in \$2. all three estimators should yield the same amount of BAO information. However, in reality, fitting ranges are finite.

We investigate the effects of this by shifting the acoustic feature in the linear theory $\omega_{0}\left(r_{s}\right), P(k)$ and $\xi(r)$ by a given $\alpha$ and then running our fit algorithms to see how well we can recover this input $\alpha$. For $\omega_{0}\left(r_{s}\right)$ we fit between $r_{s}=30-210 h^{-1} \mathrm{Mpc}$ in $r_{s}$ spacings of $2.5 h^{-1} \mathrm{Mpc}$; for $P(k)$ we fit between $k=0.0-1.2 h \mathrm{Mpc}^{-1}$ in $\log (k)$ spacings of $\sim 0.002$; and for $\xi(r)$ we fit between $r=20$ $200 h^{-1} \mathrm{Mpc}$ in $r$ spacings of $1 h^{-1} \mathrm{Mpc}$. We use the same fitting technique as described in $\$ 3.3$ but with different forms for $B(k)$ and $A(k)$ that are then transformed to $r$ and $r_{s}$ space to fit $\xi(r)$ and $\omega_{0}\left(r_{s}\right)$ respectively. This means that the fitting forms for $P(k), \xi(r)$ and $\omega_{0}\left(r_{s}\right)$ all derive from the same $B(k)$ and $A(k)$ functions. By enforcing this consistency between fitting forms, any potential systematics that may arise due to the use of different fitting forms for each statistic can be avoided.

We pick $B(k)=b$, where $b$ is the large-scale bias, and $A(k)$ to be the cold dark matter-only power spectrum multiplied by a set of cubic spline functions spec- 


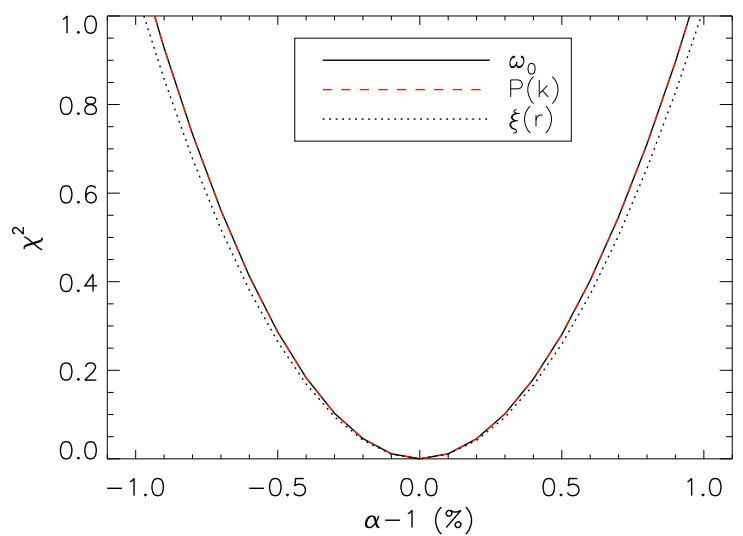

FIG. 5. - $\chi^{2}$ versus $\alpha$ for fits in $\omega_{0}\left(r_{s}\right), P(k)$ and $\xi(r)$. We shift the acoustic feature from linear theory by a given $\alpha$ (here $\left.\alpha_{\text {given }}=1.0\right)$ and run our fit algorithms to see how well we can recover this input. For $\omega_{0}\left(r_{s}\right)$ we fit between $r_{s}=30-210 h^{-1} \mathrm{Mpc}$, for $P(k)$ we fit between $k=0.0-1.2 h \mathrm{Mpc}^{-1}$ and for $\xi(r)$ we fit between $r=20-200 h^{-1} \mathrm{Mpc}$. The parabolic shape of the curves is due to the fact that $\omega_{0}\left(r_{s}\right), P(k)$ and $\xi(r)$ are derived from a Gaussian random field in linear theory. The width of the parabola at $\chi^{2}=1$ is then the theoretical $\sigma_{\alpha}$ of the fit. The $\omega_{0}\left(r_{s}\right)$ and $P(k)$ curves overlap nicely, implying that the $\sigma_{\alpha}$ ratio between $\omega_{0}\left(r_{s}\right)$ and $P(k)$ is $\sim 1$. This indicates that for the given finite fitting ranges, $\omega_{0}\left(r_{s}\right)$ and $P(k)$ contain equal amounts of acoustic information (trivially true for infinite fitting ranges by definition of $\omega_{0}\left(r_{s}\right)$, however, real surveys are finite in size). Hence, the same amount of acoustic information can be obtained through either $\omega_{0}\left(r_{s}\right)$ or $P(k)$ analysis of equal volume surveys.

ified at $k=0.01-1.09 h \mathrm{Mpc}^{-1}$. The spline points are picked so that seven of them are logarithmically spaced in the range $k=0.01-0.25$ and seven of them are linearly spaced in the range $k=0.25-1.09$ giving a total of 14 spline points. The basis functions specified by the small $k$ spline points are necessary to allow flexibility in the marginalization of $\omega_{0}\left(r_{s}\right)$ at large scales. The derivative is also specified at the first spline point to derive an additional spline function. The spline functions are taken to be natural (i.e., second derivative equal to 0 ) at the first and last spline points, beyond which linear extrapolations are implemented. This choice of $A(k)$ ensures convergence when transformed to $r_{s}$ space and makes the fits in $P(k), \xi(r)$ and $\omega_{0}\left(r_{s}\right)$ readily comparable. We assume a survey volume of $1 h^{-1} \mathrm{Gpc}$ with $\aleph=1000$ (i.e., one million particles). As a cross check, we confirmed that this new fitting form does in fact give similar results to the form used in 33.3

Figure 5 plots the $\chi^{2}$ versus $\alpha$ for $\omega_{0}\left(r_{s}\right), P(k)$ and $\xi(r)$. Here the input $\alpha$ is equal to 1 . If $\omega_{0}\left(r_{s}\right), P(k)$ and $\xi(r)$ are derived from a Gaussian random field as they are in linear theory, we would expect that $\chi^{2}$ versus $\alpha$ be parabolic as shown in the figure. The width of the parabola at $\chi^{2}=1$ is then the theoretical $\sigma_{\alpha}$ from the fit. The overlap between the $\omega_{0}\left(r_{s}\right)$ and $P(k)$ curves indicates that the ratio of $\sigma_{\alpha}$ for $\omega_{0}\left(r_{s}\right)$ to $P(k)$ is $\sim 1$. This means that for the given fitting ranges $\omega_{0}\left(r_{s}\right)$ and $P(k)$ contain equal amounts of acoustic information. Since volume is proportional to $\chi^{2}$ which is proprotional to $\sigma^{-2}$, an important implication is that we are able to obtain the same amount of acoustic information using either $\omega_{0}\left(r_{s}\right)$ or $P(k)$ analysis of equal volume surveys. We emphasize here that the results presented in this section assume idealized linear theory forms for $P(k), \xi(r)$ and $\omega_{0}\left(r_{s}\right)$. In practice, numerous physical and observational effects distort the measured statistics from these ideals. However, we expect that the features described in $\$ 2$ will reduce the impact of troublesome observational effects in any BAO analysis using $\omega_{0}\left(r_{s}\right)$.

\subsubsection{Locating the Acoustic Information}

It is useful to track down where the acoustic information lies and how it changes with $\alpha$ in $\omega_{0}\left(r_{s}\right), \xi(r)$ and $P(k)$. This is reflected in the derivatives $d \omega_{0} / d \alpha, d \xi / d \alpha$ and $d P / d \alpha$ after marginalizing out the broadband shape. To do this, we calculate the residuals from the $P(k)$ fits described in 4.2 for $\alpha=0.996$ and $\alpha=1.004$. These residuals should be representative of the acoustic signature after the broadband shape has been marginalized out. We then take $d P / d \alpha$ as the difference between these residuals divided by $1.004-0.996=0.008$. The transformations of $d P / d \alpha$ into $r_{s}$ and $r$ space then give us $d \omega_{0} / d \alpha$ and $d \xi / d \alpha$ respectively. We have plotted $d P / d \alpha$ in the top panel of Figure 6$] d \xi / d \alpha$ in the middle panel and $d \omega_{0} / d \alpha$ in the bottom panel. If one plots the ratio of $d P / d \alpha$ to $P(k) / k$, one is left with the approximate shape of the signal-to-noise ratio (SNR). This is plotted in the top right-hand corner of the top panel in Figure 6 One can see that the SNR is small at $k<0.05 h \mathrm{Mpc}^{-1}$, indicating that the small $k$ ringing in $d P / d \alpha$ is merely noise from the spline basis functions attempting to match the shape of $P(k)$ at these scales, and is not indicative of the shift in acoustic information with $\alpha$. The shifting of the acoustic information with $\alpha$ is only truly evident at $k>0.05 h \mathrm{Mpc}^{-1}$ where the SNR is larger. It is evident from these plots that the acoustic information is not as localized in $\omega_{0}\left(r_{s}\right)$ as in $\xi(r)$, but it is still reasonably well localized. The bottom panel of Figure 6 indicates that the optimal fitting range that will include all of the acoustic information encoded in $\omega_{0}\left(r_{s}\right)$ is somewhere within the range $r_{s}=30-300 h^{-1} \mathrm{Mpc}$.

The top panel of Figure 7 shows how $\sigma_{\alpha}$ changes as the minimum $r_{s}$ of the fitting range is stepped up from 30$80 h^{-1} \mathrm{Mpc}$ with the maximum $r_{s}$ of the fitting range fixed at $300 h^{-1} \mathrm{Mpc}$. The bottom panel of Figure 7 shows how $\sigma_{\alpha}$ changes as the maximum $r_{s}$ of the fitting range is stepped down from $300-120 h^{-1} \mathrm{Mpc}$ with the minimum $r_{s}$ of the fitting range fixed at $30 h^{-1} \mathrm{Mpc}$. The regions of the plots where $\sigma_{\alpha}$ begins to modulate are blown up for clarity. One can see that when the minimum of the fitting range is larger than $40 h^{-1} \mathrm{Mpc}, \sigma_{\alpha}$ begins to deviate, indicative of missing some of the acoustic information. This also happens when the maximum of the fitting range is smaller than $200 h^{-1}$ Mpc. Hence, the minimum fitting range that allows one to extract all of the acoustic information appears to be $r_{s}=40$ $200 h^{-1} \mathrm{Mpc}$. Hence, to ensure we are encapsulating all of the acoustic information, we picked the fitting range to be $r_{s}=30-210 h^{-1} \mathrm{Mpc}$ in Figure 5 .

\section{CONCLUSIONS}

We have presented a new statistic $\omega_{\ell}\left(r_{s}\right)$ for analyzing baryon acoustic oscillations. This new statistic is

7 The noise term $\sigma_{P}=P(k) / \sqrt{d N}$ where $d N=k^{2} d k$ is the number of modes out to $k$. For constant increments in $k, d N \propto k^{2}$ and hence $\sigma_{P} \propto P(k) / k$ 

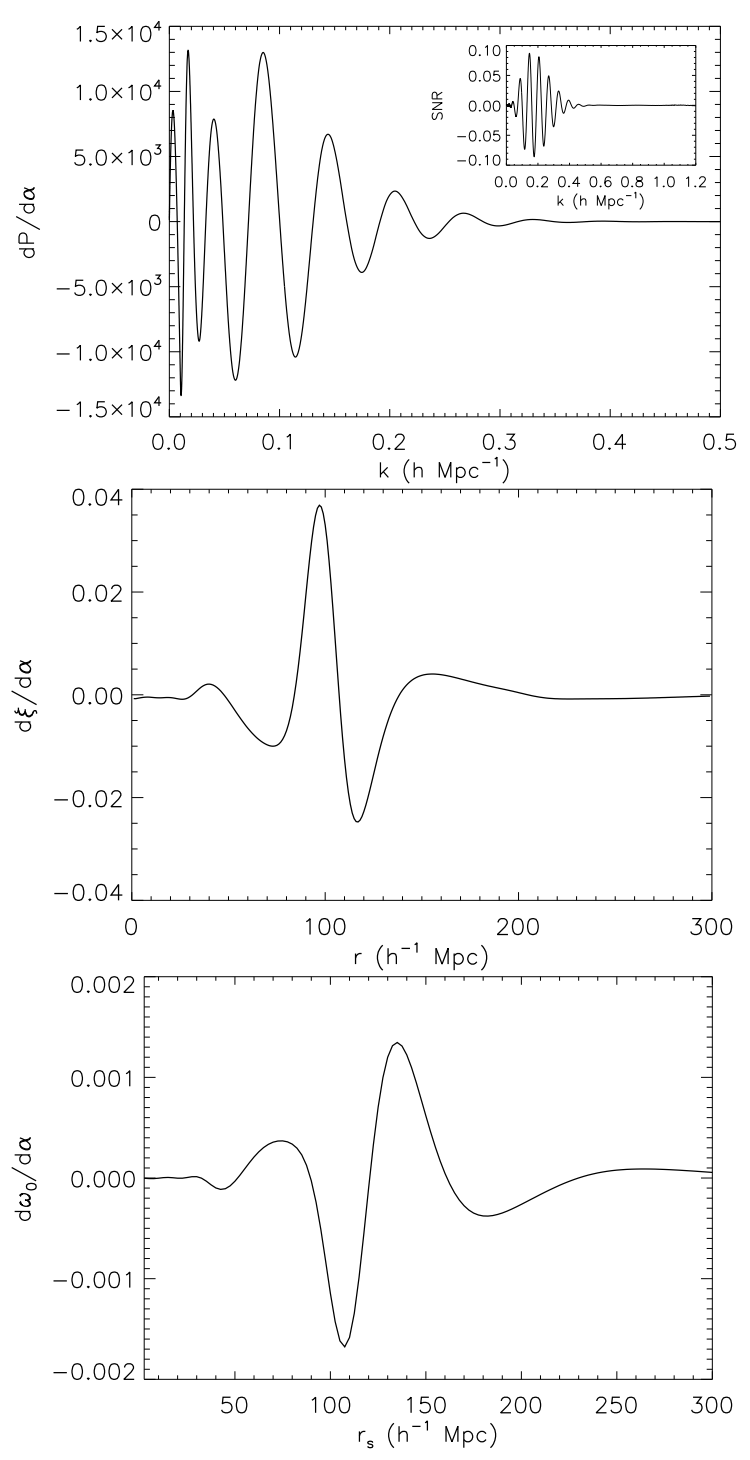

Fig. 6.- (top) $d P / d \alpha$ calculated from the residuals of the $P(k)$ fits after marginalizing out the broadband shape. The change in $P(k)$ with $\alpha$ captured by $d P / d \alpha$ should correspond to how the acoustic information is shifted as $\alpha$ changes. The ratio of $d P / d \alpha$ to $P(k) / k$ approximates the shape of the signal-to-noise ratio and is shown in the top right-hand corner of the plot. The fact that the ratio is very small at $k<0.05 h \mathrm{Mpc}^{-1}$ indicates that all the ringing in $d P / d \alpha$ at these scales is just noise from the spline basis functions attempting to match the broadband shape of $P(k)$ at these scales. Hence, this small $k$ ringing does not actually reflect the shifting of acoustic information as $\alpha$ changes. The ratio is larger for $k>0.05 h \mathrm{Mpc}^{-1}$ indicating that the oscillations in $d P / d \alpha$ at these $k$ truly reflect the shifting of acoustic information with $\alpha$. (middle) $d \xi / d \alpha$ obtained by transforming $d P / d \alpha$. This shows where the acoustic information is located in configuration space and how it changes with $\alpha$. (bottom) $d \omega_{0} / d \alpha$ obtained by transforming $d P / d \alpha$ to $r_{s}$ space. This shows where the acoustic information is located in $r_{s}$ space and how it changes with $\alpha$. Comparison with the middle panel indicates that the acoustic information is not as localized in $\omega_{0}\left(r_{s}\right)$ as it is in $\xi(r)$, however, it is still reasonably well localized. All of the acoustic information is located within $r_{s} \sim 30-300 h^{-1} \mathrm{Mpc}$, indicating that the optimal fitting range for $\omega_{0}\left(r_{s}\right)$ is somewhere within these limits.
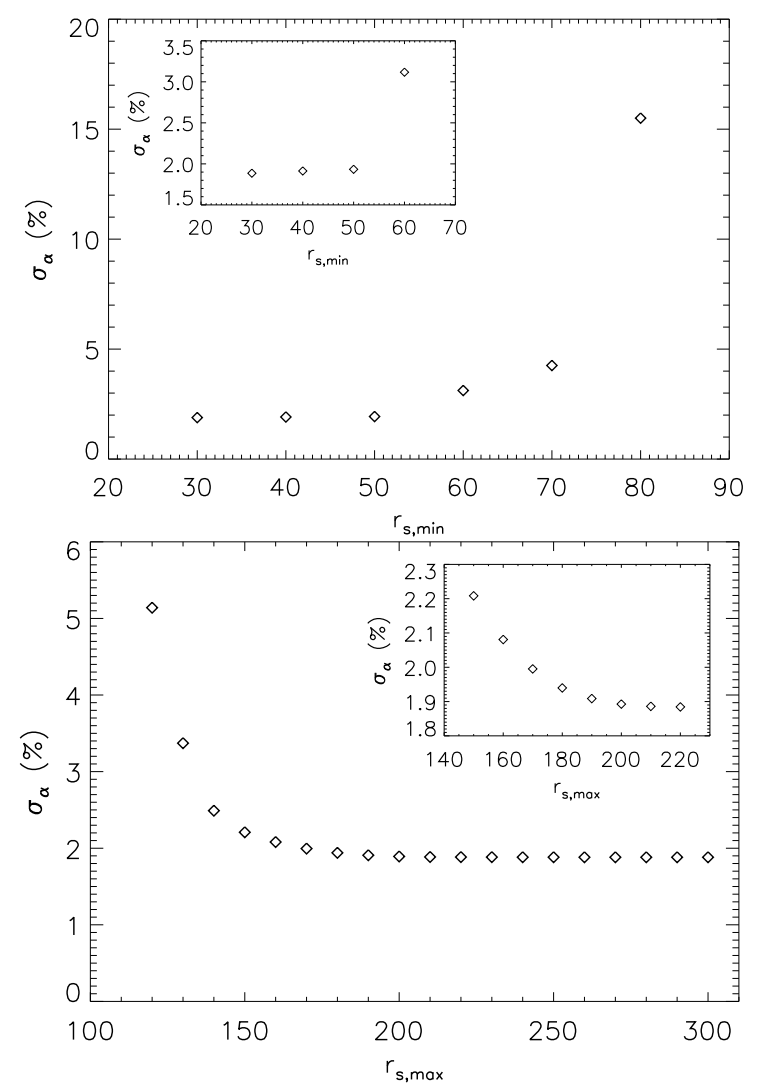

FIG. 7.- (top) Plots how $\sigma_{\alpha}$ changes as the minimum $r_{s}$ of the fitting range is stepped up from $30-80 h^{-1} \mathrm{Mpc}$ with the maximum $r_{s}$ of the fitting range fixed at $300 h^{-1} \mathrm{Mpc}$. The deviation of $\sigma_{\alpha}$ at $r_{s, \text { min }}$ larger than $40 h^{-1} \mathrm{Mpc}$ indicates that some of the acoustic information is being missed by these fitting ranges. (bottom) Plots how $\sigma_{\alpha}$ changes as the maximum $r_{s}$ of the fitting range is stepped down from 300-120h-1 Mpc with the minimum $r_{s}$ of the fitting range fixed at $30 h^{-1} \mathrm{Mpc}$. The deviation of $\sigma_{\alpha}$ at $r_{s, \max }$ smaller than $200 h^{-1} \mathrm{Mpc}$ indicates that some of the acoustic information is being missed by these fitting ranges. The above analysis implies that in order to extract all of the acoustic information, one needs to fit between $r_{s}=40-200 h^{-1} \mathrm{Mpc}$ at minimum.

advantageous over the traditional methods used to estimate $\xi(r)$ and $P(k)$ as it does away with many of their setbacks. Estimators of $\xi(r)$ are sensitive to poorly measured large scale power through effects such as the integral constraint, whereas the compensated nature of the filter $W_{\ell}(r)$ used to compute $\omega_{\ell}\left(r_{s}\right)$ circumvents this problem. We expect that this feature of the filter will also make $\omega_{\ell}\left(r_{s}\right)$ measured in different subvolumes of a survey more independent which makes error estimation methods such as bootstrap and jackknife more robust. Estimators of $P(k)$ give the true density field convolved with a window function making the measured statistic biased. Attempting to deconvolve the window function introduces artificial smoothing at small separations in $k$ due to their near delta function shapes in real observational surveys. $\omega_{\ell}\left(r_{s}\right)$, on the other hand, does not suffer from this as it is a pair count statistic. Pair counting estimators allow us to record the relative locations of tracer pairs as well as their angles from the LOS direction. This provides us with an accurate map of the underlying galaxy distribution. The fact that LOS angle is recorded also means that any anisotropic clustering should be apparent in $\omega_{\ell}\left(r_{s}\right)$. Hence we expect that it 
can also be used to probe the underlying cosmology. In addition, there is less need to worry about binning related issues when computing $\omega_{\ell}\left(r_{s}\right)$ as we never need to bin the data. The smoothness of the filter in configuration space causes the rapid fall-off of the filter in Fourier space. This reduces the impact of large $k$ modes or small scales which are not well constrained in large cosmology surveys. The localized nature of $W_{\ell}(r)$ is conducive to minimal smearing of the acoustic information so that it is mostly concentrated in a single dip around the acoustic scale. This translates to a cleaner representation of the acoustic information when plotted, as opposed to the oscillatory features of $P(k)$.

We also showed that with the present form for $W_{\ell}(r)$ and a finite fitting range encompassing the acoustic scale, it is possible to extract the same amount of acoustic information using either $\omega_{0}\left(r_{s}\right)$ or $P(k)$ from equal volume surveys. It is important to note that these results were obtained through analysis of idealized linear theory forms for $P(k), \xi(r)$ and $\omega_{0}\left(r_{s}\right)$. In practice, the measured forms of these statistics are distorted by various physical and observational effects. However, we expect that the features described in $\$ 2$ will reduce the impact of troublesome observational effects in any BAO analysis using $\omega_{0}\left(r_{s}\right)$. We also demonstrated where the acoustic information is located in $\omega_{0}\left(r_{s}\right), \xi(r)$ and $P(k)$ and how it changes with $\alpha$. From this analysis, the minimum fitting range required to extract all of the acoustic information from $\omega_{0}\left(r_{s}\right)$ appears to be $r_{s}=40-200 h^{-1} \mathrm{Mpc}$.

We compared the acoustic peak shifts derived using $\omega_{0}\left(r_{s}\right)$ to those derived using $P(k)$ for a pure DM case as well as for three halo based galaxy models. The results for the DM and the higher number density cases are all much better than $1 \sigma$ consistent with each other. The low number density case is slightly deviant with $1.6 \sigma$ agreement between $\omega_{0}\left(r_{s}\right)$ and $P(k)$. This may be a result of approaching the shot-noise limited regime or our lack of understanding of shot-noise in general and how it may affect $P(k)$ and $\omega_{0}\left(r_{s}\right)$ differently. It may also be caused by our fitting form not handling the scale-dependence of high-bias models in full. The general consistency betwen $\omega_{0}\left(r_{s}\right)$ and $P(k)$ is encouraging and implies that distance measures will be consistent between the two methods. From this and the features listed above, we conclude that $\omega_{0}\left(r_{s}\right)$ is a well-tuned new statistic for BAO analysis.

This work is supported by NASA BEFS NNX07AH11G and NSF AST-0707725. H.-J. Seo is supported by the U.S. Department of Energy under contract No. DE-AC02-07CH11359.

\section{REFERENCES}

Alcock, C., \& Paczynski, B. 1979, Nature, 281, 358

Amendola, L., Quercellini, C., Giallongo, E. 2005, MNRAS, 357, 429

Angulo, R., et al. 2008, MNRAS, 383, 755

Baumgart, D. J., \& Fry, J. N. 1991, ApJ, 375, 25

Berlind, A. A., et al. 2003, ApJ, 593, 1

Blake, C., \& Glazebrook, K. 2003, ApJ, 594, 665

Blanchard, A., \& Alimini, J.-M. 1998, A\&A, 203, L1

Bond, J. R., \& Efstathiou, G. 1984, ApJ, 594, 665

Cole, S., Fisher, K. B., Weinberg, D. H. 1994, MNRAS, 267, 785

Cole, S., Fisher, K. B., Weinberg, D. H. 1995, MNRAS, 275, 515

Crocce, M., \& Scoccimarro, R. 2006, Phys. Rev. D, 73, 063520

Crocce, M., \& Scoccimarro, R. 2008, Phys. Rev. D, 77, 023533

Davis, M., \& Peebles, P. J. E. 1983, ApJ, 267, 465

Davis, M., Efstathiou, G., Frenk, C. S., White, S. D. M. 1985, ApJ, 292, 371

de Lapparent, V., Geller, M. J., Huchra, J. P. 1988, ApJ, 332, 44

Eisenstein, D. J., \& Hu, W. 1998, ApJ, 496, 605

Eisenstein, D. J., Hu, W., Tegmark, M. 1998, ApJ, 504, L57

Eisenstein, D.J., 2003, in ASP Conference Series, volume 280

Next Generation Wide Field Multi-Object Spectroscopy, ed. M.J.I. Brown \& A. Dey (ASP: San Francisco) pp. 35-43; astro-ph/0301623

Eisenstein, D. J., et al. 2005, ApJ, 633, 560

Eisenstein, D. J., Seo, H.-J., \& White, M. 2007, ApJ, 664, 660

Feldman, H. A., Kaiser, N., Peacock, J. A. 1994, ApJ, 426, 23

Guzik, J., \& Seljak, U. 2002, MNRAS, 335, 311

Hamilton, A. J. S. 1992, ApJ, 385, L5

Hamilton, A. J. S. 1993, ApJ, 417, 19

Hamilton, A. J. S. 1998, in The Evolving Universe, ed. D. Hamilton (Dordrecht: Kluwer), 185

Hawkins, E., et al. 2003, MNRAS, 346, 78

Heavens, A. F., \& Taylor, A. N. 1995, MNRAS, 275, 483

Hewett, P. C. 1982, MNRAS, 201, 867

Holtzman, J. A. 1989, ApJS, 71, 1

Hu, W., \& Haiman, Z. 2003, Phys. Rev. D, 68, 063004

Hu, W., \& Sugiyama, N. 1996, ApJ, 471, 542

Hu, W., \& White, M. 1996, ApJ, 471, 30

Huff, E., et al. 2007, Astroparticle Physics, 26, 351

Jain, B., \& Bertschinger, E. 1994, ApJ, 431, 495

Kaiser, N. 1987, MNRAS, 227, 1

Kaiser, N., \& Peacock, J. A. 1991, ApJ, 379, 482

Komatsu, E., et al. 2009. ApJS, 180, 330

Kravtsov, A. V., et al. 2004, ApJ, 609, 35

Landy, S. D., \& Szalay, A. S. 1993, ApJ, 412, 64

Linder, E. V. 2003, Phys. Rev. D, 68, 083504
Ma, Z. 2007, ApJ, 665, 887

Matsubara, T. 2004, ApJ, 615, 573

Matsubara, T. 2007, Phys. Rev. D, 77, 063530

Mehta, K., et al. in prep

Meiskin, A., \& White, M. 1999, MNRAS, 308, 1179

Meiksin, A., White, M., Peacock, J. A. 1999, MNRAS, 304, 851

Metchnik, M., \& Pinto, P. 2010, in prep

Okumura, T., et al. 2008, ApJ, 676, 889

Padmanabhan, N., White, M., Eisenstein D.J. 2007, MNRAS, 376, 1702

Padmanabhan, N., \& White, M. 2008, Phys. Rev. D, 77, 123540

Padmanabhan, N., \& White, M. 2009, Phys. Rev. D, 80, 063508

Padmanabhan, N., et al. 2009, MNRAS, 397, 1862

Park, C., Gott, J. R., da Costa, L. N. 1992, ApJ, 392, L51

Peacock, J. A., \& Nicholson, D. 1991, MNRAS, 253, 307

Peacock, J. A., et al. 2001, Nature, 410, 169

Peebles, P. J. E., \& Yu, J. T. 1970, ApJ, 162, 815

Peebles, P. J. E. 1973, ApJ, 185, 413

Peebles, P. J. E. 1980, The Large-Scale Structure of the Universe (Princeton: Princeton University Press)

Percival, W. J., et al. 2004, MNRAS, 353, 1201

Percival, W. J., \& White, M. 2009, MNRAS, 393, 29

Sanchez, A. G., Baugh, C. M., Angulo, R. 2008, 390, 1470

Scocciamarro, R., Zaldarriaga, M. Hui, L. 1999 ApJ, 527, 1

Seo, H.-J., \& Eisenstein, D. J. 2003, ApJ, 598, 720

Seo, H.-J., \& Eisenstein, D. J. 2005, ApJ, 633, 575

Seo, H.-J., Siegel, E. R., Eisenstein, D. J., White, M. 2008, ApJ, 686,13

Seo, H.-J., et al. 2010, ApJ, submitted (astro-ph/0910.5005v2)

Sharp, N. A. 1979, A\&A, 74, 308

Sirko, E. 2005, ApJ, 634, 728

Smith, R. E., Scoccimarro, R., Sheth, R. K. 2008, Phys. Rev. D, 77,043525

Song, Y.-S. \& Percival, W. J. 2008, Phys. Rev. D, in press (astro-ph/0807.0810)

Sunyaev, R. A., \& Zeldovich, Y. B. 1970, Ap\&SS, 7, 3

Szalay, A. S., Matsubara, T., Landy, S. D. 1998, ApJ, 498, L1

Szapudi, I. 2004, ApJ, 614, 51

Tegmark, M., et al. 2006, Phys. Rev. D, 74, 123507

Tegmark, M., Hamilton, A. J. S., Xu, Y. 2002, MNRAS, 335, 887

White, M.. Song, Y.-S., Percival, W. J. 2009, MNRAS, 397, 1348

Zaroubi, S. \& Hoffman, Y. 1995, ApJ, 449, 446

Zehavi, I., et al. 2002, ApJ, 571, 172

Zehavi, I., et al. 2005, ApJ, 621, 22

Zheng, Z., et al. 2005, ApJ, 633, 791 
TABLE 1

HOD PROPERTIES

\begin{tabular}{|c|c|c|c|c|c|c|c|}
\hline Model & $\begin{array}{c}\text { Total \# } \\
\text { of Galaxies }\end{array}$ & $\begin{array}{c}\text { Satellite } \\
\text { Fraction }(\%)\end{array}$ & $\begin{array}{c}M_{\text {cen }} \\
\left(h^{-1} M_{\odot}\right)\end{array}$ & $\begin{array}{c}M_{\text {sat }} \\
\left(h^{-1} M_{\odot}\right)\end{array}$ & $\begin{array}{c}\bar{n} \mathrm{~b} \\
\left(h^{3} \mathrm{Mpc}^{-3}\right)\end{array}$ & $\bar{n} P_{0.2}$ & $\aleph_{n o n l i n}$ \\
\hline $\mathrm{DM}$ & $4 \times 10^{6}$ & - & - & - & 0.004 & 4.60 & 0.0 \\
\hline HOD1 & $2 \times 10^{6}$ & 5 & $1.4 \times 10^{12}$ & $9.2 \times 10^{13}$ & 0.002 & 5.78 & 450.0 \\
\hline HOD2 & $1 \times 10^{6}$ & 5 & $2.6 \times 10^{12}$ & $1.5 \times 10^{14}$ & 0.001 & 3.59 & 700.0 \\
\hline HOD3 & $3 \times 10^{5}$ & 5 & $6.4 \times 10^{12}$ & $3.1 \times 10^{14}$ & 0.0003 & 1.59 & 1550.0 \\
\hline
\end{tabular}

Note. - HODs are referred to by the designations under the "Model" heading throughout the paper.

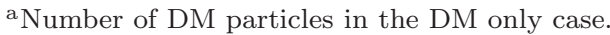

${ }^{\mathrm{b}}$ The nominal Poisson shot-noise is $\bar{n}^{-1}$.

TABLE 2

Fit RESUlts FOR EACH HOD MODEL

\begin{tabular}{cccccccc}
\hline Model & $\begin{array}{c}\alpha-1 \\
(\%)\end{array}$ & $\begin{array}{c}\sigma_{\alpha} \\
(\%)\end{array}$ & $\Sigma_{n l}$ & $r_{\text {scale }}$ & $\begin{array}{c}\chi^{2} \\
\text { (per d.o.f) }\end{array}$ & $\begin{array}{c}\text { bias }^{\text {a }} \text { (b) } \\
\sigma_{8}\end{array}$ & $\sigma_{8}$ \\
\hline DM & 0.0457 & 0.2333 & 6.66 & 19.99 & 0.92 & 1.25 & 0.63 \\
HOD1 & 0.1065 & 0.2243 & 5.61 & 19.78 & 0.94 & 2.04 & 1.11 \\
HOD2 & 0.1634 & 0.2449 & 5.85 & 19.98 & 0.86 & 2.28 & 1.25 \\
HOD3 & 0.4897 & 0.3326 & 6.27 & 20.04 & 0.72 & 2.77 & 1.55 \\
\hline
\end{tabular}

Note. - Fitting range: $30 \leq r_{s} \leq 200 h^{-1}$ Mpc. $\sigma_{\alpha}$ is the error on the mean $\alpha$ of the 44 simulations.

${ }^{\text {a }}$ Bias is not equal to 1 for the DM only case because we are working in redshift space. 
TABLE 3

DIFFERENCE IN MEAN $\alpha$ BETWEEN $\omega_{0}\left(r_{s}\right)$ AND $P(k)$

\begin{tabular}{cc}
\hline Model & $\left\langle\Delta \alpha_{\omega P}\right\rangle$ \\
& $(\%)$ \\
\hline DM & $0.0516 \pm 0.1205$ \\
HOD1 & $0.0076 \pm 0.0672$ \\
HOD2 & $0.0205 \pm 0.0600$ \\
HOD3 & $0.1035 \pm 0.0665$ \\
\hline
\end{tabular}

APPENDIX

\section{EVALUATING $\widetilde{W}_{\ell}(K)$}

The expressions for $\widetilde{W}_{\ell}(k)$, in terms of polynomials of $k$ times trigonometric functions, involve a lot of cancellation. This makes them unstable to direct evaluation. However if we define

$$
K_{n}(k)=\frac{2+n}{\left(k r_{s}\right)^{2+n}} \int_{0}^{k r_{s}} x^{n} \sin x d x
$$

then

$$
\widetilde{W}_{0}(k)=\frac{8 \pi}{3}\left[\frac{1}{3}\left(K_{7}-K_{16}\right)-\left(K_{10}-K_{13}\right)\right]
$$

while

$$
\widetilde{W}_{2}(k)=-\frac{24 \pi}{5 k^{2}}\left[3 K_{5}-16 K_{8}+25 K_{11}-12 K_{14}\right] .
$$

It is straightforward to evaluate $K_{n}(x)$, the limits are

$$
K_{n}(x)=1-\frac{n+2}{3 !(n+4)} x^{2}+\frac{n+2}{5 !(n+6)} x^{4}+\cdots
$$

as $x \rightarrow 0$ and

$$
K_{n}(x)=-(n+2) \frac{\cos x}{x^{2}}+n(n+2) \frac{\sin x}{x^{3}}+\cdots
$$

as $x \rightarrow \infty$. The $K_{n}$ also satisfy a simple recurrence relation

$$
K_{n}(x)=\frac{n+2}{x^{3}}\left[n \sin x-x \cos x-(n-1) x K_{n-2}\right] .
$$

Use of this recurrence relation for high $k$ and the power-series expansion for low $k$ results in stable evaluation of the $\widetilde{W}_{\ell}$. 